\title{
Image Contrast Enhancement for Deep-sea Observation Systems
}

\author{
Huimin $\mathrm{Lu}^{\mathrm{a}, \mathrm{b}, \mathrm{c}, \mathrm{d}^{*}}$, Yujie $\mathrm{Li}^{\mathrm{b}}$, Jianru $\mathrm{Li}^{\mathrm{c}}$, Xin $\mathrm{Li}^{\mathrm{d}}$, Yohei Kashio ${ }^{\mathrm{b}}$, Seiichi Serikawa ${ }^{\mathrm{b}}$ \\ aJapan Society for the Promotion of Science, 102-0083 Tokyo, Japan \\ ${ }^{b}$ Kyushu Institute of Technology, 804-8550 Kitakyushu, Japan \\ 'State Key Laboratory of Marine Geology, Tongji University, 200092 Shanghai, China \\ dNational Laboratory of Naval Architecture and Ocean Engineering, \\ Shanghai Jiaotong University, 200240 Shanghai, China
}

*Corresponding Author: luhuimin@ ieee.org

\begin{abstract}
This paper describes a novel method for enhancing ocean optical images. While light traveling through the water, light rays are distorted depending on the wavelength. That is, absorption, scattering and color distortion are three major distortion issues for underwater optical imaging. In this paper, our key contributions are proposed include a novel deep-sea imaging model to compensate for the attenuation discrepancy along the propagation path and an effective underwater scene enhancement scheme. The recovered images are characterized by a reduced noised level, better exposure of the dark regions, and improved global contrast where the finest details and edges are enhanced significantly. The experiments show that the average value of PSNR and SSIM is improved than the state-of-the-art-methods.
\end{abstract}

Keywords: deep-sea imaging, inherent optical properties, image reconstruction.

\section{Introduction}

With the development of exploring the deep-sea by autonomous underwater vehicles (AUVs) and unmanned underwater vehicles (UUVs), the resolution of underwater images remains as a major issue. That is, how to acquire a clear underwater image is a question. From the 1960s, sonar has been widely used for detection and recognition of objects in oceans. Because of acoustic imaging principle, the sonar imaged images have many shortcomings, such as the low signal to ratio, low resolution et al. Consequently, optical vision sensors must then be used instead for short-range identification because of the low quality of images restored by sonar imaging [1].

In contrast to normal images, underwater images suffer from poor visibility owing to the medium. The light is absorbed when sunlight is reflected by a water surface. Furthermore, light is also deflected and scattered by many particles in water. In addition, absorption substantially reduces the atmospheric light energy. The random attenuation of light primarily causes a hazy appearance, while the fraction of light scattered back from the water along the line of sight considerably degrades the scene contrast. In particular, objects at a distance of more than 10 meters are almost indistinguishable, because the colors are faded owing to the characteristic wavelengths that are filtered according to the water depth [2]. Furthermore, a distinctive footprint of the light beam is typically left on the seafloor when an artificial light source is employed.

In the last decade, there are some researchers focused on underwater image quality improvement. Y.Y. Schechner et al. exploited a polarization imaging method to compensate for visibility degradation [3], while Ancuti et al. used an image fusion method in a turbid medium to reconstruct a clear image [4]. Hou et al. combined a point spread function and modulation transfer function to reduce the effects of blurring [5]. Ouyang et al. proposed bilateral filtering based on an image deconvolution method [6]. Although the aforementioned approaches can enhance the image contrast, these methods have demonstrated several drawbacks that reduce their practical applicability. First, the equipment for imaging is difficult to use in practice (e.g., a range-gated laser imaging system, which is hardly applied in practice). Second, multiple input images are required [7]. Third, they cannot alleviate color distortion very well.

Instead of multiple input images, underwater scene reconstruction methods using a single image have been proposed. The ICA based dahazing method was first proposed by Fattal [8]. He estimated the scene radiance and derived the transmission image by a single image. However, this method cannot sufficiently process images with heavy haze. Then, He et al. [9] proposed a scene depth information-based dark channel prior dehazing algorithm using a matting Laplacian. However, this algorithm requires significant computation time. To overcome this disadvantage, they also proposed a new guided image filter [10] with the foggy image used as a reference image. However, this method leads to incomplete haze removal. 
Hence, in this paper, we introduce a novel approach that is able to enhance underwater images based on a single image to overcome the drawbacks of the conventional methods mentioned above. The organization of this paper is as follows. Section 2 explains the ocean imaging model. Section 3 describes the model for underwater image enhancement and proposes our guided trigonometric bilateral filter. Section 4 applies our proposed method to underwater optical images. Finally, Section 5 concludes this paper.

\section{Underwater Imaging Model}

Artificial light and atmospheric light traveling through the water is the source of illumination in an ocean environment. Let suppose the amount of radiation light $W(x)$ formed after wavelength attenuation can be formulated according to the energy attenuation model as follows:

$$
\begin{aligned}
& E_{\lambda}^{W}(x)=E_{\lambda}^{A}(x) \cdot \operatorname{Nrer}(\lambda)^{D(x)}+E_{\lambda}^{I}(x) \cdot \operatorname{Nrer}(\lambda)^{L(x)}, \\
& \quad \lambda \in\{r, g, b\}
\end{aligned}
$$

where $E_{\lambda}^{W}(x)$ is the amount of illumination at point $x$, $E_{\lambda}^{A}(x)$ is the amount of illumination of atmospheric light at point $x, E_{\lambda}^{I}(x)$ is the illumination of artificial light, and Nrer is the normalized residual energy ratio. At the scene point $x$, artificial light reflected again travels distance $L(\mathrm{x})$ to the camera forming pixel $I_{\lambda}(x), \lambda \in\{r, g, b\} . D(x)$ is the scene depth underwater. We suppose the absorption and scattering rate is $\rho(x)$, artificial light $J_{\lambda}(x)$ emanated from point $x$ is equal to the amount of illuminating ambient light $E_{\lambda}^{W}(x)$ reflected,

$$
\begin{aligned}
& E_{\lambda}^{W}(x)=\left(E_{\lambda}^{A}(x) \cdot \operatorname{Nrer}(\lambda)^{D(x)}+E_{\lambda}^{I}(x) \cdot \operatorname{Nrer}(\lambda)^{L(x)}\right) \cdot \rho(x), \\
& \lambda \in\{r, g, b\}
\end{aligned}
$$

By following the improved Nayar-Narasimhan hazing model [13], the image $I_{\lambda}(x)$ formed at the camera plane can be formulated as,

$$
\begin{aligned}
I_{\lambda}(x)= & \left(E_{\lambda}^{A}(x) \cdot \operatorname{Nrer}(\lambda)^{D(x)}+E_{\lambda}^{I}(x) \cdot \operatorname{Nrer}(\lambda)^{L(x)}\right) \cdot t_{\lambda}(x) \\
& +\left(1-t_{\lambda}(x)\right) \cdot B_{\lambda}, \quad \lambda \in\{r, g, b\}
\end{aligned}
$$

where the background $B_{\lambda}$ represents the part of the object reflected light $J_{\lambda}$, ambient light $E_{\lambda}^{W}$ scattered toward the camera by particles in the water. The residual energy ratio $t_{\lambda}(x)$ can be represented alternatively as the energy of a light beam with wavelength $\lambda$ before and after traveling distance $d(x)$ within the water $E_{\lambda}^{\text {residual }}(x)$ and $E_{\lambda}^{\text {initial }}(x)$, respectively, as follows:

$$
t_{\lambda}(x)=\frac{E_{\lambda}^{\text {residual }}(x)}{E_{\lambda}^{\text {initial }}(x)}=10^{-\beta(\lambda) d(x)}=\operatorname{Nrer}(\lambda)^{d(x)}
$$

where Nrer is the normalized residual energy ratio [14], in the Ocean Type I, it follows:

$$
N_{\text {rer }}(\lambda)=\left\{\begin{array}{cl}
0.8 \sim 0.85 & \text { if } \lambda=650 \sim 750 \mu m(\text { red }) \\
0.93 \sim 0.97 & \text { if } \lambda=490 \sim 550 \mu m(\text { green }) \\
0.95 \sim 0.99 & \text { if } \lambda=400 \sim 490 \mu m(\text { blue })
\end{array}\right.
$$

Consequently, subscribing the Eq. (3) and Eq. (4), we can obtain:

$$
\begin{aligned}
I_{\lambda}(x)= & {\left[\left(E_{\lambda}^{A}(x) \cdot \operatorname{Nrer}(\lambda)^{D(x)}+E_{\lambda}^{I}(x) \cdot \operatorname{Nrer}(\lambda)^{L(x)}\right) \cdot \rho_{\lambda}(x)\right] \cdot \operatorname{Nrer}(\lambda)^{d(x)} } \\
& +\left(1-\operatorname{Nrer}(\lambda)^{d(x)}\right) \cdot B_{\lambda}, \quad \lambda \in\{r, g, b\}
\end{aligned}
$$

The above equation incorporates the light scattering during the course of propagation from object to the camera $d(x)$, and the wavelength attenuation along both the light-object path $L(x)$, scene depth $D(x)$ and object-camera path $d(x)$. Once the light-object distance $L(x)$, scene depth $D(x)$ and object-camera distance $d(x)$ is known, the final clean image will be recovered.

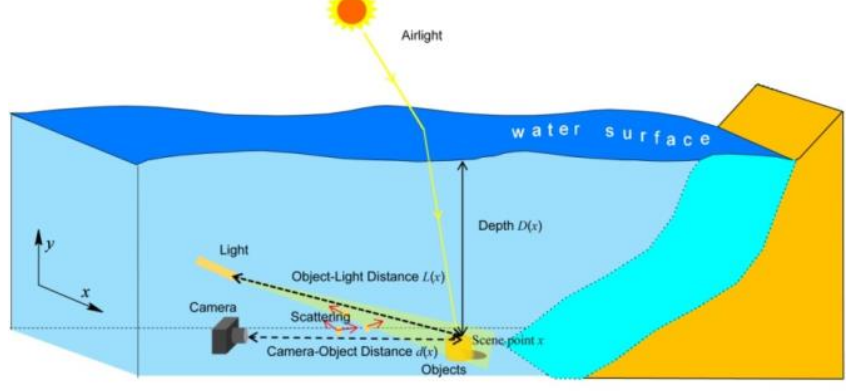

Figure 1. Diagram of Shallow Ocean Optical Imaging Model.

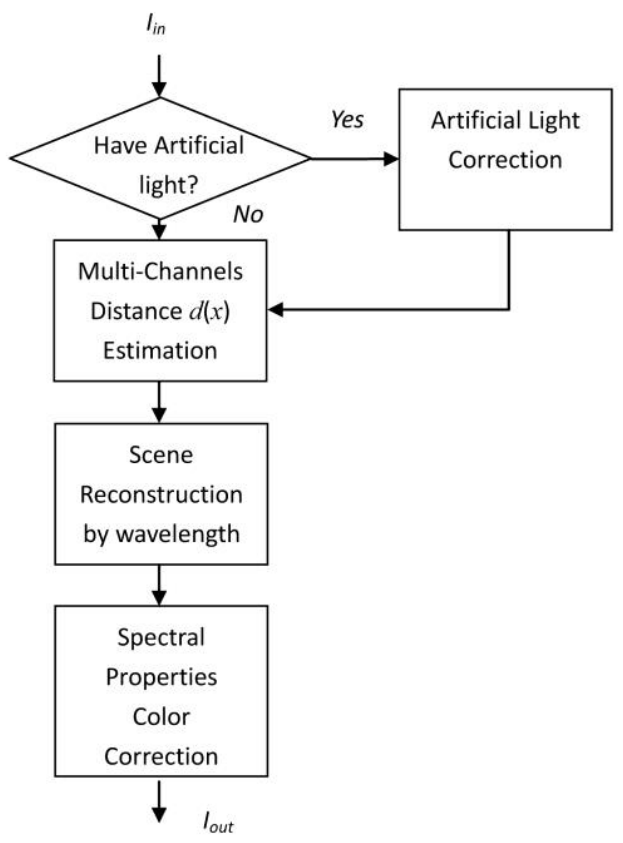

Figure 2. Pipeline of video frame improvement method

Figure 1 shows the diagrammatic sketch of the proposed model. For improving the image quality, we take the processing flowchart as Figure 2.

\section{Underwater Image Improvement Methods}




\subsection{Artificial Light Adjustment}

In the deep sea, we must use artificial light for imaging. However, it will cause the vignetting effect. In ref. [28], K. Sooknanan et al. proposed a multi-frame vignetting correction model for removing the vignetting phenomenon which involves estimating the light source footprint on the seafloor. This artificial light correction can well done, however, it cost large time for computing. So, in this paper, we intend to introduce a signal frame-based vignette removal method [29].

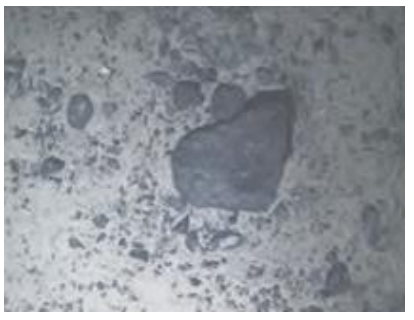

(a)

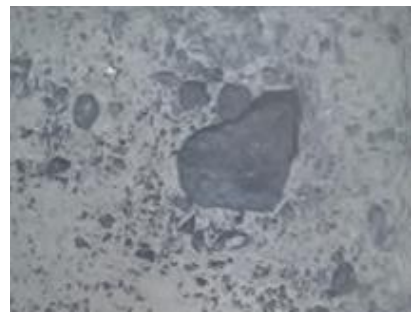

(b)
Figure 3. Simulation result of artificial light correction. (a) Input image; (b) Artificial light adjusted image.

\subsection{Absorption and Scattering Removal}

In Ref. [19], the author found the red color channel is the dark channel of underwater images. During our experiments, we found that the lowest channel of RGB channels in turbidly water is not always the red color channel; the blue color channel is very significant $[25,27]$. The reason is that we usually take artificial light in imaging. Although the red wavelength absorbed easily through traveling in water, the distance between the camera and object is not enough to absorb the red wavelength significantly. The blue channel may be the lowest. Consequently, in this paper, we take the minimum pixel value as the rough depth disparity.

We propose a guided trigonometric bilateral filter (GTBF) to overcome the occurrence of gradient reversal artifacts. The filtering process of the GTBF is first performed under the guidance of image $G$ that can be another image or the input image $I$ itself. Let $I_{p}$ and $G_{p}$ be the intensity value at the pixel $p$ of the minimum channel image and the guided image, respectively, and $w_{k}$ be the kernel window centered at pixel $k$, consistent with the bilateral filter. GTBF is then formulated by,

$$
\operatorname{GTBF}(I)_{p}=\frac{1}{\sum_{q \in w_{k}} W_{G T B F_{p q}}(G)} \sum_{q \in w_{k}} W_{G T B F_{p q}}(G) I_{q},
$$

where the kernel weight function $W(G)$ can be written by

$$
W_{G T B F_{p q}}(G)=\frac{1}{|w|^{2}} \sum_{k:(p, q) \in w_{k}}\left(1+\frac{\left(G_{p}-\mu_{k}\right)\left(G_{q}-\mu_{k}\right)}{\sigma_{k}^{2}+\varepsilon}\right),
$$

where $\mu_{k}$ and $\sigma_{k}^{2}$ are the mean and variance of the guided image $G$ in the local window $w_{k}$, and $|w|$ is the number of pixels in this window. When both $G_{p}$ and $G_{q}$ are concurrently on the same side of an edge, the weight assigned to pixel $q$ is large. When $G_{p}$ and $G_{q}$ are on different sides, a small weight will be assigned to pixel $q$.

As we obtained the refined depth disparity $d(x)$, in order to remove the scatter, we also need to solve the reflectivity $\rho_{\lambda}(x)$. We take the least squares solution for achieving this by [19] as,

$$
\begin{aligned}
& \rho_{\lambda}(x)=\left(J_{\lambda}(x)^{T} \cdot J_{\lambda}(x)\right)^{-1} \cdot J_{\lambda}(x)^{T} \\
& \quad \cdot\left(E_{\lambda}^{A}(x) \cdot \operatorname{Nrer}(\lambda)^{D(x)}+E_{\lambda}^{I}(x) \cdot \operatorname{Nrer}(\lambda)^{L(x)}\right), \\
& \quad \lambda \in\{r, g, b\}
\end{aligned}
$$

After removing the artificial light, the Eq. (6) can be written as

$$
\begin{aligned}
I_{\lambda}(x)= & E_{\lambda}^{A}(x) \cdot \operatorname{Nrer}(\lambda)^{D(x)} \cdot \rho_{\lambda}(x) \cdot \operatorname{Nrer}(\lambda)^{d(x)} \\
& +\left(1-\operatorname{Nrer}(\lambda)^{d(x)}\right) \cdot B_{\lambda}, \quad \lambda \in\{r, g, b\}
\end{aligned}
$$

According to Nayar-Narasimhan hazing model, we can obtain the descattered image by

$$
\begin{aligned}
\tilde{J}_{\lambda}(x) & =\frac{I_{\lambda}(x)-\left(1-\operatorname{Nrer}(\lambda)^{d(x)}\right) \cdot B_{\lambda}}{\operatorname{Nrer}(\lambda)^{d(x)}} \\
& =E_{\lambda}^{A}(x) \cdot \operatorname{Nrer}(\lambda)^{D(x)} \cdot \rho_{\lambda}(x) \cdot \operatorname{Nrer}(\lambda)^{d(x)}, \\
& \lambda \in\{r, g, b\}
\end{aligned}
$$

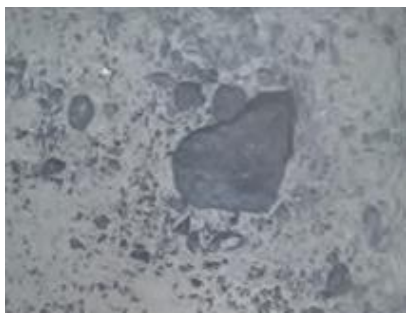

(a)

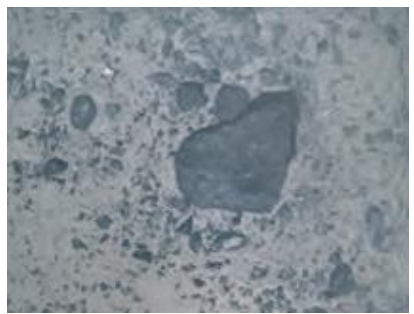

(b)

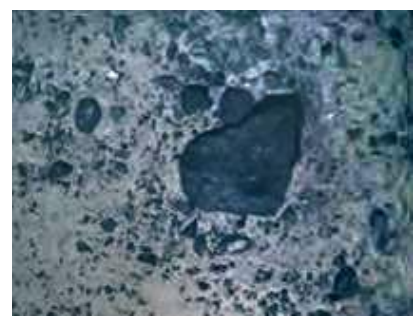

(c)

Figure 4. Simulation result of absorption and scattering removal. (a) Artificial light adjusted image; (b) Absorption corrected result; (c) De-scattered result.

\subsection{Color Correction}

In Ref. [19], the author simply corrected the scene color by the attenuation of water depth. However, in practice, the spectral response function [30] of a camera maps the relative sensitivity of the camera imaging system as a function of the wavelength of the light. We take the chromatic transfer function $\tau$ for weighting the light from the surface to a given depth of objects as

$$
\tau_{\lambda}=\frac{E_{\lambda}^{\text {surface }}}{E_{\lambda}^{\text {object }}}
$$

where the transfer function $\tau$ at wavelength $\lambda$ is derived from the irradiance of the surface $E_{\lambda}^{\text {sufface }}$ by the irradiance 
of the object $E_{\lambda}^{\text {object }}$. Based on the spectral response of RGB camera, we convert the transfer function to RGB domain:

$$
\tau_{R G B}=\int_{\lambda=400}^{\lambda=725} \tau_{\lambda} \cdot C_{b}(\lambda)
$$

where the weighted RGB transfer function is $\tau_{R G B}, C_{b}(\lambda)$ is the underwater spectral characteristic function of color band $b, b \in\{r, g, b\}$.

Finally, the corrected image as gathered from the weighted RGB transfer function by

$$
J_{\lambda}(x)=\hat{J}_{\lambda}(x) \cdot \tau_{R G B}
$$

where $J_{\lambda}(x)$ and $\hat{J}_{\lambda}(x)$ are the color corrected and uncorrected images, respectively.

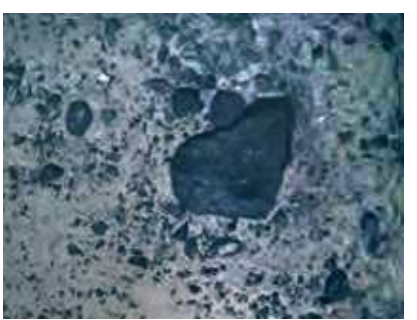

(a)

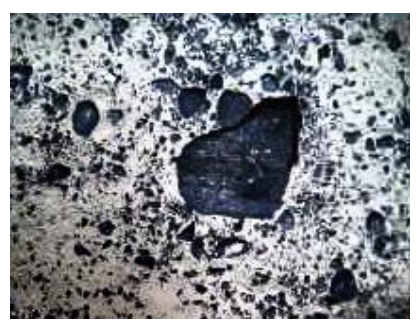

(b)
Figure 5. Simulation result of absorption and scattering removal. (a)

De-scattered image; (b) Color corrected result.

\section{Experiments and Discussions}

The performance of the proposed algorithm is evaluated both objectively and subjectively by utilizing ground-truth color patches. Both results demonstrate superior haze removal and color balancing capabilities of the proposed method over the others. In the experiment, we compare our method with Schechner's model, Bazeille's model, Fattal's model, Nicholas's model; He's model, Xiao's model, Ancuti's model, and Chiang's model. Here, we select the best parameters for each model. The computer used is equipped with Windows XP and an Intel Core 2 (2.0 $\mathrm{GHz}$ ) with 1 GB RAM.

Figure 6 shows the results using different reconstruction methods. The size of the images is $600 \times 424$ pixels. The processed image of Bazeille's pre-processing method is serious distorted. The drawback of Fattal's method is obviously, which is because it needs to manually determine the background and foreground in the image. It is hard to use in practical application. While Nicholas's Graph-cut based method cost a lot of processing time, while the processed image is also blurred. The right corner of the processed image is seriously distorted. In comparison with He's method, our approach performs better, and as visible mosaic artifacts are observed in He's approach owing to the use of soft matting. Some of the regions are too dark (e.g. the right corner of the coral), and haze is not removed elsewhere (e.g. the center of the image). The contrast of Xiao's model is not strong. In addition, there are

also some unresolved scatters or haze-like objects around the coral reefs in Ancuti's model. How to select the parameters for fusion is a hard work. Moreover, Chiang's work is distorted in colors. Our proposed method takes the guided trigonometric bilateral filter and spectral properties based color correction method to recover the distorted underwater images, which are shown superior to the state-of-the-art methods both on computation complex and visual effects. Consequently, compare with the above methods, our proposed method is the best.

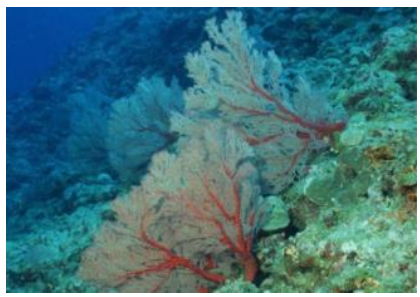

(a)

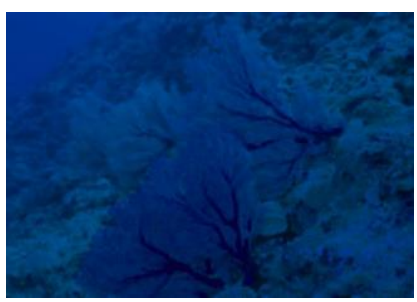

(c)

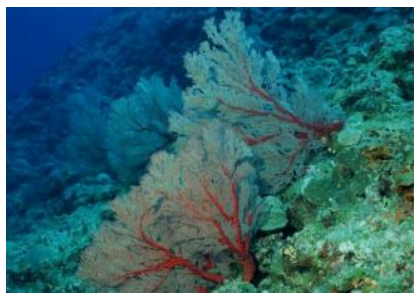

(e)

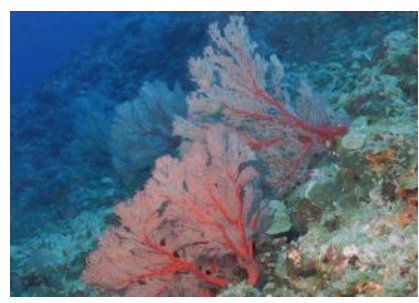

(g)

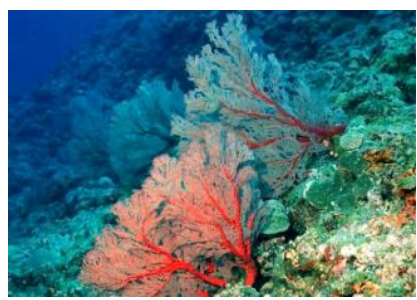

(i)

Figure 6. Different models for underwater coral image reconstruction: (a)

Input image; (b) Bazeille's model; (c) Fattal's model; (d) Nicholas's model; (e) He's model; (f) Xiao's model; (g) Ancuti's model; (h) Chiang's

model; and (i) our proposed model. (b)

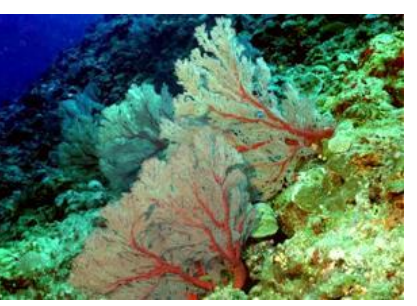

(d)

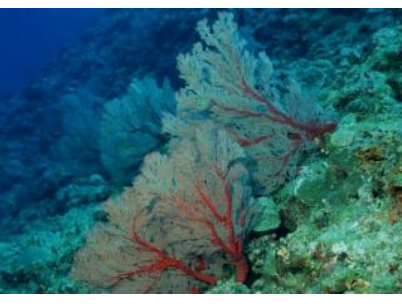

(f)

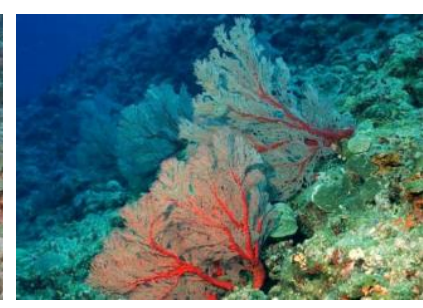

(h) 
In addition to the visual analysis mentioned above, we conducted quantitative analysis, mainly from the perspective of mathematical statistics and the statistical parameters for the images (see Table 1). This analysis includes Peak Signal to Noise Ratio (PSNR) [22], Quality mean-opinion-score (Q-MOS) [18], and Structural SIMilarity (SSIM) [23]. Table 1 displays the numerical results of Q-MOS, PSNR and SSIM measured on several images. The results indicate that our approach works well for haze removal.

Table 1. Comparative Analysis of Different De-scattering Metho
\begin{tabular}{c|c|c|c|}
\hline Methods & $P S N R$ & $Q-M O S$ & $S S I M$ \\
\hline Bazeille & 13.0844 & 19.8556 & 0.2061 \\
\hline Fattal & 11.7316 & 19.2204 & 0.4581 \\
\hline Nicholas & 18.9630 & 43.0762 & 0.7763 \\
\hline He & 23.2120 & 77.8366 & 0.9642 \\
\hline Xiao & 24.5089 & 66.0857 & 0.9493 \\
\hline Ancuti & 27.0414 & 39.8664 & 0.9074 \\
\hline Chiang & 27.3216 & 71.7899 & 0.9597 \\
\hline Our & 27.7829 & 77.6780 & 0.9698 \\
\hline
\end{tabular}

\section{Conclusions}

In this paper, we have explored and implemented novel image enhancement techniques for shallow water optical image reconstruction. We have proposed a simple prior based on the difference in attenuation among the different color channels, which inspired us to estimate the transmission depth map. Another contribution compensated the transmission by weighted guided trigonometric bilateral filter, which has the benefits of edge-preserving, noise removing, and a reduction in the computation time. Moreover, the proposed spectral-based underwater image colorization method successfully created colorful underwater distorted images that are better than the state-of-the-art methods.

\section{Acknowledgment}

This work was partially supported by Grant in Aid for Japan Society for the Promotion of Science Fellows (No.13J10713), Grant in Aid for Non-Japanese Researchers by NEC C\&C Foundation, The Telecommunications and Advancement Foundation, State Key Laboratory of Marine Geology, Tongji University, China (MGK1407) and National Laboratory of Naval Architecture and Ocean Engineering, Shanghai Jiaotong University, China (1301). The first author wishes to thank Japan Agency for Marine-Earth Science and Technology (JAMSTEC) for offering the deep-sea images and videos.

\section{References}

(1) D.M. Kocak; F.R. Dalgleish; F.M. Caimi; Y.Y. Schechner. A focus on recent developments and trends in underwater imaging. Mar. Technol. Soc. J., 42(1), 52-67, 2008.

(2) R. Schettini; S. Corchs. Underwater image processing: state of the art of restoration and image enhancement methods. EURASIP J. Adv. Sig. Pr., 746052, 2010.

(3) Y.Y. Schechner; Y. Averbuch. Regularized image recovery in scattering media. IEEE T. Pattern Anal., 29(9), 1655-1660, 2007.

(4) C. Ancuti; C.O. Ancuti; T. Haber; P. Bekaert. Enhancing underwater images and videos by fusion. In Proceedings of IEEE Conference on Computer Vision and Pattern Recognition, 81-88, 2012.

(5) W. Hou; D.J. Gray; A.D. Weidemann; G.R. Fournier; J.L. Forand. Automated underwater image restoration and retrieval of related optical properties. In Proceedings of IEEE International Symposium of Geoscience and Remote Sensing, 1889-1892, 2007.

(6) B. Ouyang; F.R. Dalgleish; F.M. Caimi; A.K. Vuorenkoski; T.E. Giddings; J.J. Shirron. Image enhancement for underwater pulsed laser line scan imaging system. In Proceedings of SPIE, 8372, 83720R, 2012.

(7) T. Treibitz; Y.Y. Schechner. Turbid scene enhancement using multi-directional illumination fusion. IEEE T. Image Process., 21(11), 4662-4667, 2012.

(8) R. Fattal. Signal image dehazing. In SIGGRAPH, 1-9, 2008.

(9) K. He; J. Sun; X. Tang. Single image haze removal using dark channel prior. IEEE T. Pattern Anal., 33(12), 2341-2353, 2011.

(10)K. He; J. Sun; X. Tang. Guided image filtering. In Proceedings of the 11th European Conference on Computer Vision, 1, 1-14, 2010.

(11)C. Tomasi; R. Manduchi. Bilateral filtering for gray and color images. In Proceedings of IEEE International Conference on Computer Vision, 839-846, 1998.

(12)K.N. Chaudhury; D. Sage; M. Unser. Fast O(1) bilateral filtering using trigonometric range kernels. IEEE T. Image Process., 20(12), 3376-3382, 2011.

(13)A. Rizzi; C. Gatta; D. Marini. A new algorithm for unsupervised global and local color correction. Pattern Recogn. Lett., 24, 1663-1677, 2003.

(14)M. Chambah; D. Semani; A. Renouf; P. Courtellemont; A. Rizzi. Underwater color constancy: enhancement of automatic live fish recognition. In Proceedings of SPIE, 5293, 157-168, 2004.

(15) M. Bertalmio; V. Caselles; E. Provenzi; A. Rizzi. Perceptual color correction through variational techniques. IEEE $\mathrm{T}$. Image Process., 16(4), 1058-1072, 2007.

(16) J. Tarel; N. Hautiere. Fast visibility restoration from a single color or gray level image. In Proceedings of IEEE Conference of Computer Vision and Pattern Recognition, 2201-2208, 2009.

(17)C. Xiao; J. Gan. Fast image dehazing using guided joint bilateral filter. Visual Comput., 28(6-8), 713-721, 2012.

(18)R. Mantiuk; K.J. Kim; A.G. Rempel; W. Heidrich. HDR-VDP2: a calibrated visual metric for visibility and quality predictions in all luminance conditions. ACM T. Graphic., 30(4), 40-52, 2011. 
(19)J.Y. Chiang; Y.C. Chen. Underwater image enhancement by wavelength compensation and dehazing. IEEE T. Image Process., 21(4), 1756-1769, 2012.

(20)S. Bazeille; I. Quidu; L. Jaulin; J.P. Malkasse. Automatic underwater image pre-processing. In Proceedings of Caracterisation Du Milieu Marin, 1-8, 2006.

(21)C-B Nicholas; M. Anush; R.M. Eustice. Initial results in underwater single image dehazing. In Proceedings of IEEE OCEANS, 1-8, 2010.

(22)http://www.ni.com/white-paper/13306/en/

(23)Z. Wang; A. C. Bovik; H. R. Sheikh; E. P. Simoncelli. Image quality assessment: from error measurement to structural similarity. IEEE T. Image Process., 13(1), 600-612, 2004.

(24)J.M. Morel; A. B. Petro; C. Sbert. Fast implementation of color constancy algorithms. In Proceedings of SPIE, 7241, 724106, 2009.

(25)S. Serikawa; H. Lu. Underwater image dehazing using joint trilateral filter. Comput. Elec. En., 41(1), 41-50, 2014.

(26)ITU-R-BT.500-11, Methodology for the subjective assessment of the quality of television pictures, 2002.

(27)H. Lu; Y. Li; S. Serikawa. Underwater image enhancement using guided trigonometric bilateral filter and fast automatic color correction. In Proceedings of IEEE International Conference on Image Processing, 3412-3416, 2013.

(28)K. Sooknanan; A. Kokaram; D. Corrigan. Improving underwater visibility using vignetting correction. In Proceedings of SPIE, pp.83050M-1-8, 2012.

(29) Y. Zheng; S. Lin; S.B. Kang; R. Xiao; J.C. Gee; C. Kambhamettu. Single-image vignetting correction from gradient distribution symmetries. IEEE T. Pattern Anal., 35(6), pp.1480-1494, 2013.

(30)D.L. Bongiorno; M. Bryson; D.G. Dansereau; S.B. Williams. Spectral characterization of COTS RGB cameras using a linear variable edge filter. In Proceedings of SPIE-IS\&T, vol.86600N, 2013. 